\title{
An Automated Attendance System: A Technique using Background Subtraction and Color Image Processing of RGB Planes
}

\author{
Puspendra Kumar Pateriya and Sagar \\ School of Computer Science \& Engineering, Lovely Professional University, Phagwara - 144411, Punjab, India; \\ pushpendra.mnnit@gmail.com, sagarpalyal@yahoo.com
}

\begin{abstract}
Background/Objectives: The objective of this paper is to introduce an automated attendance marking system for LPU using CCTV cameras. It detects the students sitting in the class and takes the attendance automatically without any human assistance and count total number of students present in the class also. Methods/Statistical Analysis: An enhanced detection algorithm is proposed that is having two phases: color image processing of RGB planes and modified background subtraction of image which executed simultaneously for object detection. Before detection process, noise is removed from images by using log function which uniformly distributes the brightness over the image. To count the number of detected objects, a counter is used which calculates total number of objects present in an image. The Automatic ROI detection algorithm is also proposed which detects region of interest automatically on the basis of repeated regular pattern of object. Findings: The system able to count and mark attendance of students sitting in class room. Implementation shows that our proposed approach has $89.5 \%$ accuracy with less false positive and false negative rate as compared to other traditional approaches. The Automatic ROI detection algorithm also detects the region of interest with considerably high accuracy. Application/Improvements: The proposed system will easy to deploy in various schools, colleges, universities and other educational institutes with minimal setup cost where CCTV cameras are already installed in class rooms.
\end{abstract}

Keywords: Background Subtraction, Log Function, Object Detection, RGB Color Image Processing, Region of Interest

\section{Introduction}

There is a demand for the object detection and recognition techniques. In image processing and computer vision, object recognition is an important task. To recognize the objects with the help of machines, algorithmic description for the object recognition tasks must be implemented on the particular machine. So, the less complex and efficient object recognition techniques need to be developed ${ }^{1}$.

To save time and to make it easier for the user to detect and locate the objects in the digital images, various applications are implemented such as video surveillance system, car parking system etc. In digital images, detection of the target object in presence of other objects is a major problem ${ }^{2}$.

The process of recognition and identification of different instances of real world entities like humans, vehicles etc. in an image or video is known as object detection. The area of an image where the object can be located is called region of interest (ROI) ${ }^{3}$. The ROI may contain a single object or multiple objects. Algorithms of object detection generally use extracted features for recognizing the objects. Feature extractor is used to extract relevant features from the input image. These relevant features are also known as extracted features. These features can be of fundamental type or geometrical type which represents the behaviour or structure of the object respectively. Various applications of object detection algorithms are, character or digit recognition system, security surveillance system, biometric recognition system and automated vehicle parking system etc. An image may contain more than one ROI.

Counting and detection of objects having arbitrary shape such as humans in a complex scene is not an easy task. Many researchers are still working on the problem of human detection and head counting. Illumination

${ }^{*}$ Author for correspondence 
changes, shadow, color variance and brightness make the task more complex. It is also very difficult to detect an object under various rotations. So, primary need is to remove the noise in the image by any image preprocessing or image restoration technique and detect angle of the object. Finding the location of a person in an image is also a challenging task.

Occluded humans are difficult to detect and count. Thus, detecting and counting the number of persons and also finding the position for the same in a crowded scene is a hot cake in the field of research work.

$\mathrm{In}^{2}$ introduced the color processing and Circular Hough Transform (CHT). Color processing eliminated the unrelated color from the image and $\mathrm{CHT}$ detected the shape of the object by using some pre-defined radius of circular pattern. After the detection phase, they used a counter which automatically counts the total objects present in the input image.

The Haar-classifier with various features like head, eye, upper body, face, lower body etc. was used by Dharani, Gowri and Ramya for human detection in video clip. Frame differencing technique was applied for the subtraction of foreground from background on Region of Interest (ROI) $)^{3}$.

Active Contour Model (ACM) and Watershed segmentation were used by Tareque and Hasan for detection of lip contour. $\mathrm{H}$ filter and Lyapunov stability theory was introduced to track the detected lip contour. Result of tracking became more stable and accurate by introducing Lyapunov stability theory ${ }^{4}$.

In $^{5}$ worked on Active Shape Model (ASM) to extract the geometric lip features and to provide the local tracking of lip. They also used Analytic Hierarchy Process (AHP) along with Fuzzy classifier for matching the particular word against the video frame. An accuracy of $83.2 \%$ achieved as compared to other algorithms by using this approach.

In $^{6}$ introduced 8-Region Neighborhood Growing Algorithm for the detection of contour of an object and this process of finding the objects contour is termed as contour evaluation. After evaluating contour of the object, they calculated Curvature Scale Space (CSS) by plotting Gaussian value and corresponding arc length on the graph. Only those features of CSS plot were taken that were above decided threshold value. The system was robust for scaled, rotated and noisy images.

In ${ }^{7}$ worked on Level Set Method (LSM) for detecting the boundary of an image. This method did not give good and accurate results under noise. Diffusion filter was used as a noise removal from the images. Still, detection rate was improved by using the method as compared to previous methods.

$\mathrm{In}^{8}$ worked on different layers for boundary detection of any object from the input image or video frame. The layers are divided into three levels named low, mid and high. The low-level layer worked on grey level intensity or color values, mid-level layer used segmentation and optical flow for the boundary detection, high-level layer used object category segmentation for boundary detection. The accuracy of the system increased with less computation cost.

$\mathrm{In}^{9}$ worked on detection and tracking of human arm by using the method KLT named as Kanade-LucasTomasi. To get the better tracking results, they introduced the motion model named as Kalman filter that made best possible prediction of arms movement. This method was compatible for indoor environment with various object pose, different scenes and complex background.

$\mathrm{In}^{10}$ worked on line based shape features approach and canny edge technique for the detection of probability of boundaries. After the detection of boundaries, human figure was localized where high dense area $\mathrm{Pb}$ (Probability of boundary) features found. Then Hough transform fit the straight lines to detected boundaries and converted the features into histograms. They also worked on entropy based approach for action detection where high change in entropy found.

In ${ }^{11}$ proposed the adaptive filtering technique along with Bayesian change detection algorithm for edge detection and correction of moving objects respectively. The method worked well under illumination changes, shadows, noise. Also, there was no requirement of background and foreground in advance. For practical implementations, the method was very suitable and efficient.

Background subtraction with dynamic threshold was proposed by Yang, Shi and Yi for the detection of motion objects. Dynamic threshold improved the detection of moving pixels in video frames. To remove noise, the morphological processing technique was applied over the video frame or image. For complex backgrounds, the method provides good detection results while taking less computational time ${ }^{12}$.

$\mathrm{In}^{13}$ presented the comparative analysis of different techniques of image edge detection. They found that canny edge detection algorithm worked better than 
Robert, Prewitt, Sobel and LoG (Laplacian of Gaussian) algorithms but computationally expensive.

In ${ }^{14}$ proposed an approach that used object patches and relative position of these patches with the center of the object as a feature. They worked on improved gentleboost classifier to train the detector. They used two phases for object detection. First phase was learning phase and other was detection phase. In first phase, some of the images were selected randomly with certain objects, extracted the objects and trained the detector with improved gentleboost algorithm. In second phase, new input test image was detected with the trained detector of learning phase and provided the final detection results. The algorithm considered each patch of the image as foreground that means object to be detected and rest as non-object background. Gentleboost classifier provided better detection results. Efficiency and accuracy was improved. Accuracy would also improve in case of classification if they managed to include part-based detector for detection of partial occluded objects.

$\operatorname{In}{ }^{15}$ proposed an algorithm that was the combination of template matching and Fuzzy Support Vector Machine (FSVM). There were object samples as well as nonobject samples in an image, so FSVM classifier marked the position of the object and non-object samples in the original image and separated them. The combination of template matching and FSVM classifier was used for the detection of object in general scenes. Template matching algorithm classified the input samples into background and foreground. The FSVM classifier then again classified the output sample of the template matching algorithm. The template matching algorithm was parallelized by using OpenMP. The computational performance of the system improved significantly by using the algorithm as compared to traditional serial algorithms. The accuracy was improved and false alarm was reduced with the use of FSVM classifier ${ }^{15}$.

Aydin and Udur proposed a general color clustering technique named Ant Colony Optimization (ACO) algorithm. They used and tested the algorithm in a case study for the extraction of boundary of the object on the images of flower. ACO approach used the color clustering technique for color image segmentation. The method tried to determine the cluster centre points and number of color clusters, and assigned these pixels to the closest clusters. According to the color distribution in image, it merged the closest clusters. The method was efficient in color image segmentation ${ }^{16}$.
$\mathrm{In}^{17}$ worked on an algorithm named as Adaptive Targets-detecting algorithm. The algorithm comprised of Local Binary Patterns(LBP) and Background Modelling Method (BMM). For the situations of sudden brightness changes, a light information pre-treatment approach was introduced by the researchers. Firstly, texture feature was obtained by calculating LBPs and then normalized histogram of the LBP texture was plotted. After this, background model was established by using texture features. For input frame, every pixel i.e. histogram value was matched with background model. If values were matched, then area belonged to background, otherwise foreground. The algorithm showed robustness against disturbances in the background. Real-time performance, adaptability and accuracy of the detection rate in complex scenes were improved. But this approach was only applicable to static backgrounds. They combined Gaussian method with LBP to remove shadow effect ${ }^{17}$.

$\mathrm{In}^{18}$ introduced a method for the extraction of edge information named as Modified Gray Level Cooccurrence Matrix (MGLCM) and for the detection of threshold value for the classification of object from background in a blurred or low contrast images. The method calculated the average of all the pixels that was under consideration including neighborhood pixels. Due to averaging the pixel values, this method was capable of handle the noisy image. For the detection of threshold value, the algorithm used the GLCM quadrant. GLCM was divided into four quadrants. The first and the last quadrants contained the information regarding transition in gray level within the foreground or object and within the background respectively. These quadrants denoted as local quadrants. The information regarding transition between the object and the background was paced in the remaining quadrants. These quadrants denoted as joint quadrants. The algorithm worked well with low contrast and blurred images ${ }^{18}$.

In $^{19}$ worked on Circular Hough Transform (CHT), Bayesian approach and model matching for detecting and counting the crowd. In model matching, threedimensional human shape models were matched with the input image to separate the foreground from the background and calculating the probability of the object or human boundary. The primary goal of the approach was to segment the image into background as nonhuman object and foreground as human object by using a threshold technique name multiple adaptive thresholds. These foreground objects may be occluded. For the 
nomination of various candidates in the image, CHT based an upper semicircle detector was implemented. The performance of this approach as compared to the different global optimized methods showed that the approach had less computational cost. Results showed that the approach is robust but sometimes did not detect the occluded human ${ }^{19}$.

$\mathrm{In}^{20}$ worked on segmentation and closely coupled object detection algorithms. They used template matching along with these algorithms for the detection of human's upper body. They also introduced colour based and shape based segmentations. They used the weighted sum of estimations to find the probability of foreground pixels in the input image on the basis of colour based and shape based segmentations. Firstly, they initialized the foreground pixels on the basis of template matching in the input image. After finding the probability of foreground pixels, the colour based segmentation followed by the shape based segmentation was applied to the processed image and made estimation about the presence of person on the interested foreground region. The process was repeated over time for finding the stable results. The proposed method improved the detection rate of the boundary of person. The algorithm was able to detect humans from different projections. The algorithm was not able to recognize the people under occlusion. Those People who wore cloths having colour same as that of background was also not detected by the proposed algorithm ${ }^{20}$.

In $^{21}$ proposed a part based detection method for detecting and tracking of human. They represented the whole body of human as four parts: head-shoulder, torso, legs and full body. The object was tracked on the basis of body parts, thus, making the algorithm worked under occlusion. They used the data association and mean shift tracker for the tracking of the detection object. The proposed method also worked under rotation. Once the part based detector detected the object, the trajectory was initialized. For the initialization of trajectory, the combined response of detection was used having low rate of false alarm. Initialization of trajectory could work well under inter-object occlusion but not under scene occlusion. All part responses were used by the combined tracker for the tracking of detected object. Tracking of object worked under both inter-object occlusion and scene occlusion. For the growth of trajectory, the data association technique was used. If it was failed to track the object, then mean shift tracker came into play for the tracking of object. Trajectory was terminated if there was no response come from object for a particular period of time ${ }^{21}$.

Reddy et al. proposed some parameters for detection of changes in the video frames that are not recognized by the naked eye. These parameters are Signal to Noise ratio, Normalized Correlation, Absolute Average Difference, Mean Squared Error and Peak Signal to Noise ratio. These parameters analyze each pixel value of corresponding pixels of two frames. If these changes are perceived by human eye, then these parameters show greater difference in values. The result shows that proposed method able to detect even small changes that are not visible to human eye and it will be helpful in video analysis ${ }^{22}$.

$\mathrm{In}^{23}$ introduced fuzzy expert system for the skin color detection of humans with the help of rule based or knowledge based information of expert. Skin is the combination of RGB color space in color images. The proposed system able to identify the different intensity of skin color based upon some fuzzy rules. These intensities are high, median, low and very low. The system was able to detect the skin of humans with an accuracy of $80 \%{ }^{23}$.

\section{Problem Formulation}

In the era of advanced technology, everything is changing from the manual system to the automated system having some kind of artificial intelligence which astonishes the people time to time. From security to surveillance, sports to gaming, medical to astronomy, in every field the intelligent system already established their roots.

But in every schools and colleges, why we still use the old methods of taking the attendance by manually marking the present or absent of the students. Although, with the advancement of new technologies the attendance system changed from offline mode to online mode, but still there is no kind of any intelligence in the system. Teachers or Lectures are bound to mark the present or absent manually even in the online attendance system. There are some negative view points of manually marking the attendance. It is a time consuming task if there are 40-50 students in a class. There is always the possibility of marking the wrong attendance by the human which is very common problem with manually marking the attendance.

To reduce such a burden from the shoulders of human being, this report proposed the new attendance system 
having some kind of artificial intelligence which will automatically takes the attendance of the students sitting in the class-room. It will also increase the performance and decrease the time interval for taking the attendance even if the strength of the class lies between 40-50 or more than fifty. Performance will increase in the sense that there will be no chance of marking the wrong attendance and also, student will not be able to shout the proxy for their friends. The new attendance system will automatically recognize the students sitting on the bench and mark them presents and others will be marked absent.

\section{Methodology of our Approach}

The proposed system so far consists of three phases: pre- processing phase, detection phase and counting phase. The first phase uses log function for pre-processing of images. This function reduces intensity value of pixels where the intensity is high and vice-versa. Thus, it makes uniform intensity over the images. The second phase detects presence of object based upon two approaches: background subtraction and color image processing. If the result of both approaches is positive, then object is present otherwise not. Accuracy of our proposed system increases by merging the results of background subtraction and color image processing. The last phase counts the total number of objects on the basis of their presence or absence in the input image. The data flow chart of our proposed approach is shown in Figure 1.

The proposed approach is to make an attendance

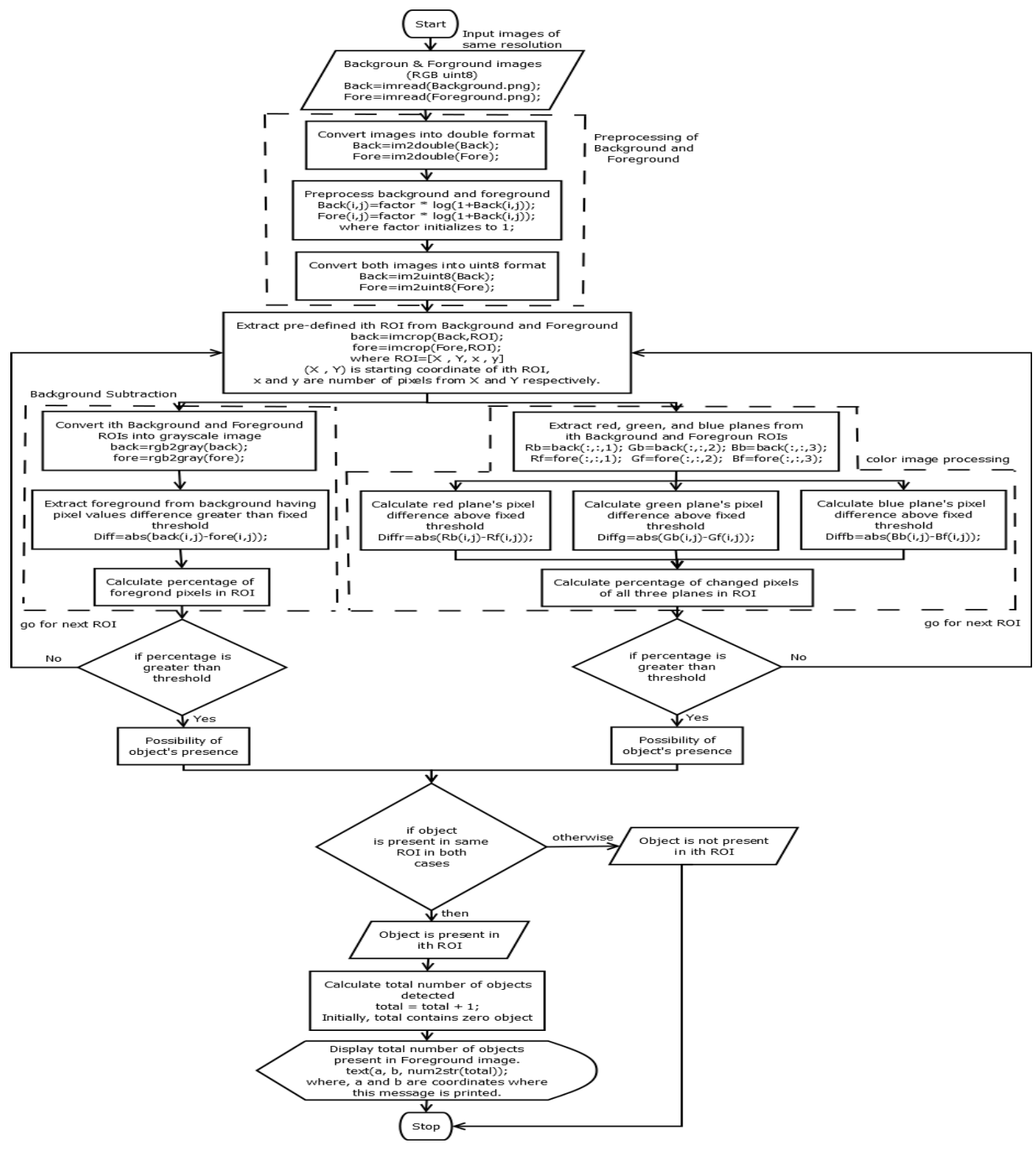

Figure 1. Data flow chart of proposed approach. 
system which automatically detects presence or absence of students sitting in the class and marks attendance accordingly. The whole process can be broken down as follows:

- Input background and foreground images.

- Pre-process both images for removing noise.

- Extract pre-defined ROIs from background and foreground images.

- Perform color image processing of RGB planes and background subtraction over each ROI side by side.

- If both approaches show positive result, then object is present in our region of interest otherwise not.

- Finally, total number of objects present in foreground image is counted by using a counter.

Automatic ROI detection algorithm is also proposed which automatically detects chairs inside a room based upon repeated regular pattern of black color of chairs. Firstly, the algorithm inverts the image so that all the black pixels convert into white pixels. After inversion, the image is transformed into binary image to convert all the non-white pixels into black pixels. Now the region having connected white pixels more than fixed threshold is our region of interest. The algorithm detects this pattern and stores the coordinated of detected ROIs into array named 'ROI'. If detected coordinate is already a part of predetected ROI then eliminate it from the array 'ROI'.

The proposed detection algorithm and automatic ROI detection algorithm are shown in Algo1 and Algo2 respectively.

Algorithm 1 Proposed detection algorithm.

1. Input Background and Foreground RGB images by using Matlab inbuilt function imread.

2. Pre-process both images by using log function.

3. Create and Initialize pre-defined ROI for designated places.

4. Loop

a. Color processing of RGB planes for each ROI.

b. Background subtraction of each ROI.

c. Merge results of color processing and background subtraction for corresponding ROIs. If object is detected in both cases only then final merged result shows detection of object.

d. Print rectangle over the detected objects that are found after merging color processing and background subtraction.

5. End Loop
6. Count the total number of detected objects by using a counter.

7. Display total objects detected over the Foreground image.

Algorithm 2 Automatic ROI detection algorithm

1. Input a RGB image by using Matlab inbuilt function imread.

2. Invert the input image.

3. Convert the image into grayscale format.

4. Convert the grayscale image into binary format.

5. Remove the noise from image by using median filter.

6. Create array ROI for storing the coordinates of detected objects.

7. Loop

a. Store coordinates of first white pixel after every black pixel.

b. Calculate total number of connected white pixels and store it into variable count.

c. If black pixel is encountered, initialize count to zero.

d. Eliminate ROIs having coordinates in the range of previously detected ROIs.

e. If number of connected white pixels is greater than threshold value, then store the coordinates into array ROI.

8. End Loop

9. Prints the ROIs over the detected objects.

\section{Results and Discussion}

To perform implementation, hp Pavillion g6 Notebook PC has used with Intel(R) Core(TM) i3-2350M CPU@2.30GHz Processor, 6 GB RAM, 64-bit Operating System. Code has implemented and executed over the MATLAB platform with version 8.1.0.604 (R2013a) having licence number 724504 and image processing toolbox version 8.2.

The dataset of different class rooms has taken from the Lovely Professional University. Due to wrong CCTV camera locations or some objects come in front of these cameras like fan, we could not able to perform our implementation on every room. We take couple of rooms with perfect camera locations and did our implementation over different frames. The data set have very complex images and also due to low resolution of CCTV cameras, we restrict our research work to certain region. The right part of class room is excluded from 
our research work because of less clarity of region even to human eye. Some bottom part of class room is also excluded because it comes under some printed text which could be an overhead during our implementation part.

The process of automatic ROI detection using algorithm is shown in Figure2. We are using fixed size dimensions for each ROI. But the ratio of size of chairs is changing sharply. Due to this overhead few numbers of ROIs are not detected by our approach. So, pre-defined ROIs are used in proposed detection algorithm because we are not getting 100\% accuracy in automatic ROI detection.
With our proposed approach, we are able to detect the objects as well as count total number of detected objects in indoor environment. We are able to achieve accuracy around $90 \%$ for object detection. Comparison of our approach with other traditional techniques like Background Subtraction and color image processing shows that our approach yields good detection results.

When any object is present in the foreground frame, it drastically affects the histogram values. Figure 3 and Figure 4 shows histogram values without object and with object respectively. These histograms are taken from two different ROIs of color image processing of frame

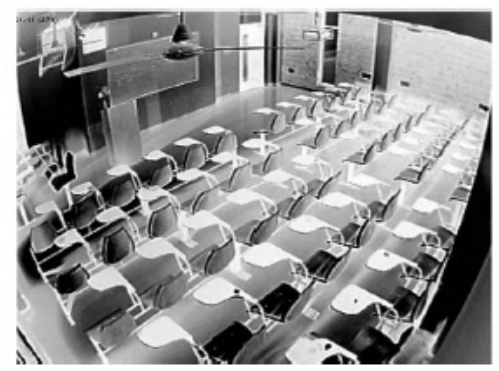

(b)

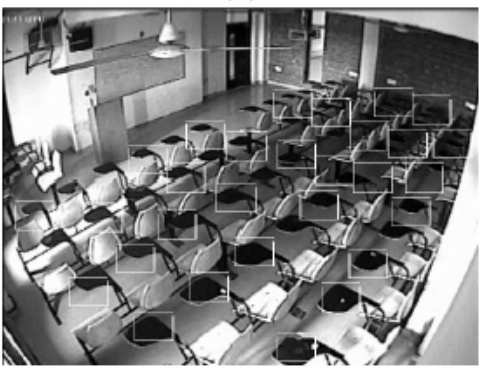

(d)

Figure 2. Process of automatic ROI detection based on repeated black color chair pattern of room number 34-507 (a) Input image. (b) Invert image and convert into grayscale. (c) Convert into binary image. (d) Detection of ROI.

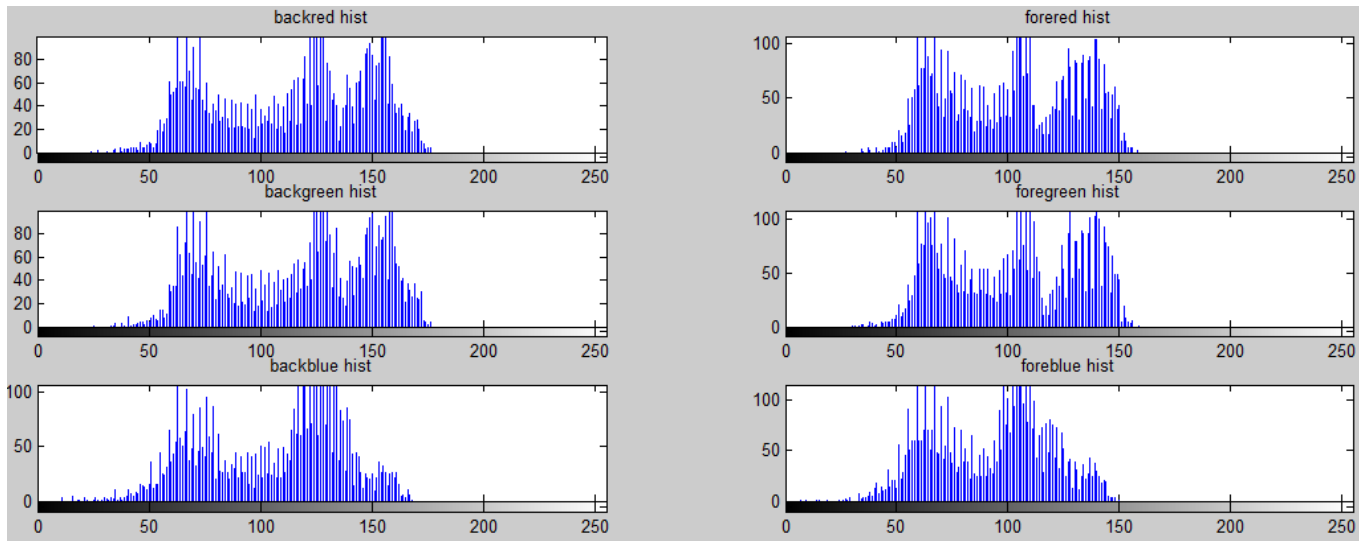

Figure 3. Histogram value without object. 


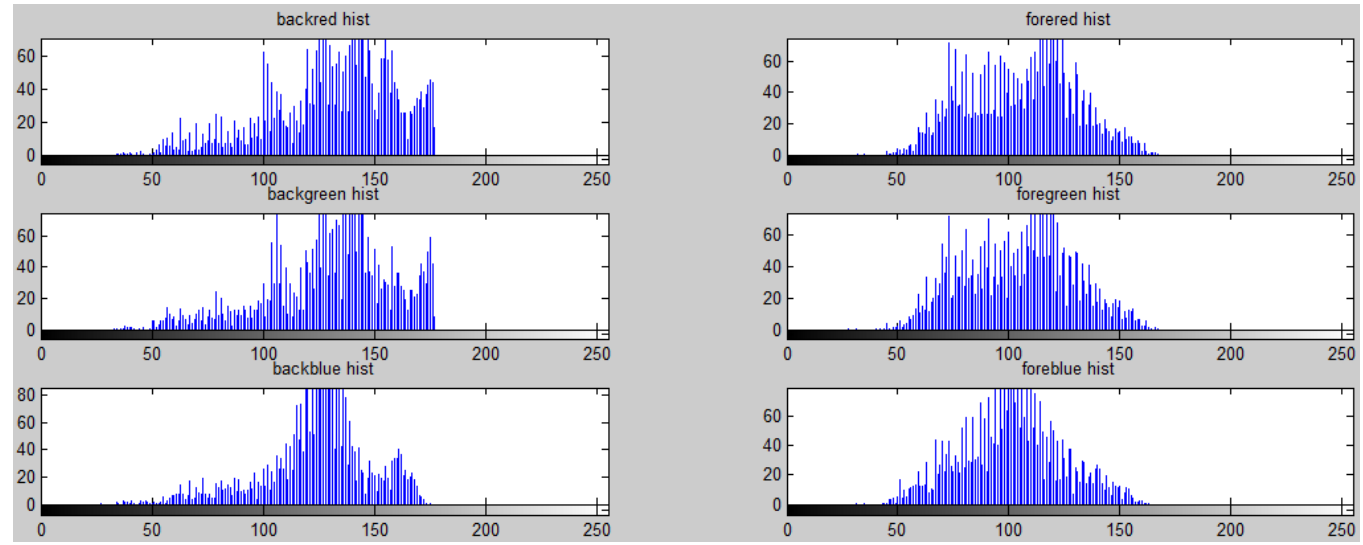

Figure 4. Histogram value with object.

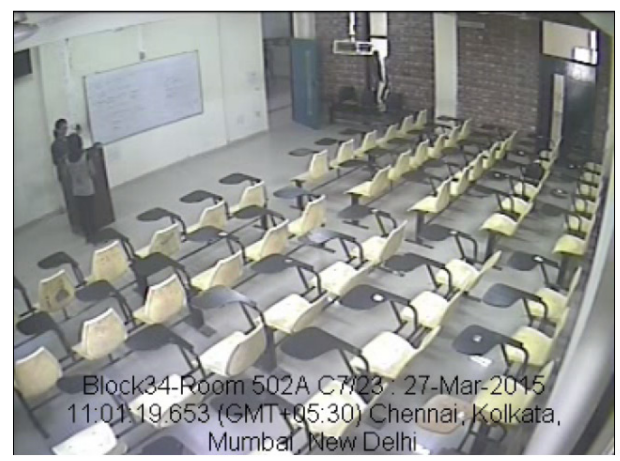

(a)

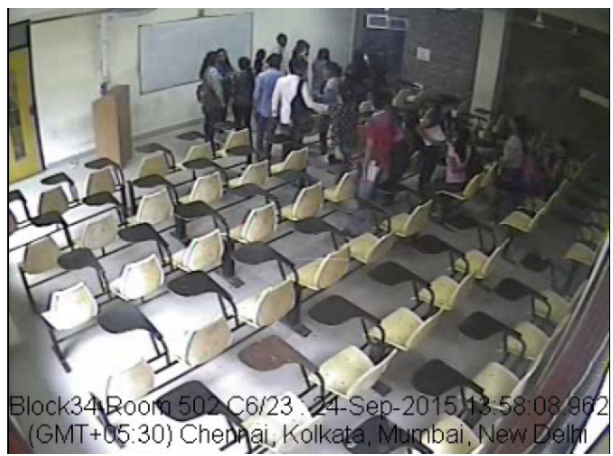

(b)

Figure 5. Background images of class rooms (a) Class room 34-502A. (b) Class room 34502.

34-502A of class room 34-502A. It is easily interpreted that histogram values of background and foreground are different when object is in the frame.

The background images of different class rooms are shown in Figure 5. The results of proposed detection algorithm on different frames of class room 34-502A and 34-502 are shown in Figure 6 and Figure 7 respectively. Comparison of our proposed approach with other techniques like color processing of RGB planes and traditional background subtraction shows that our approach has more accuracy and less false positive rate. False negative rate of our approach and color processing have same values but less than traditional background subtraction.

Implementation results of our proposed approach, color processing and background subtraction on class rooms 34-502A and 34-502 are shown in Tables 1, 2 and 3 respectively.
Accuracy of detection results is calculated by using the Accuracy $=\frac{T P-F P-F N}{T P}$

where, TP is Total Places or ROIs in the image, FP and FN are False Positive and False Negative rate respectively. Some other abbreviations used in this paper are:

- TP: Total number of places we are interested in or ROIs (Region of Interests).

- APS: Actual number of present students in the class.

- DS: Total number of detected students after executing detection algorithm.

- FN: False negative means object is present but it is not detected by detection algorithm.

- FP: False positive means object is not present in frame but results shows that object is present or wrong detection. 

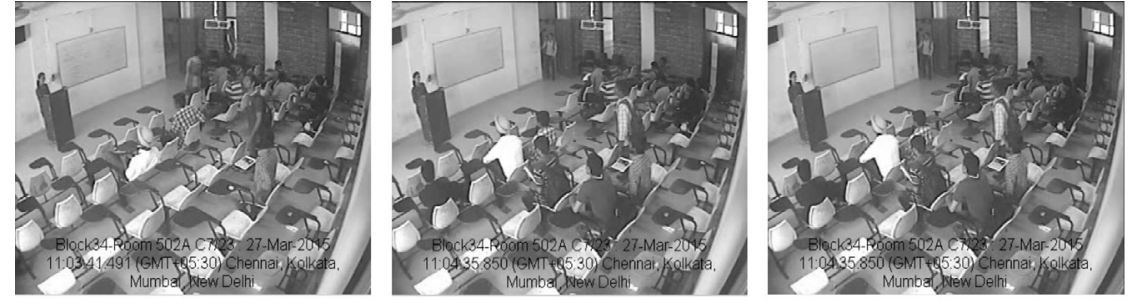

(a)
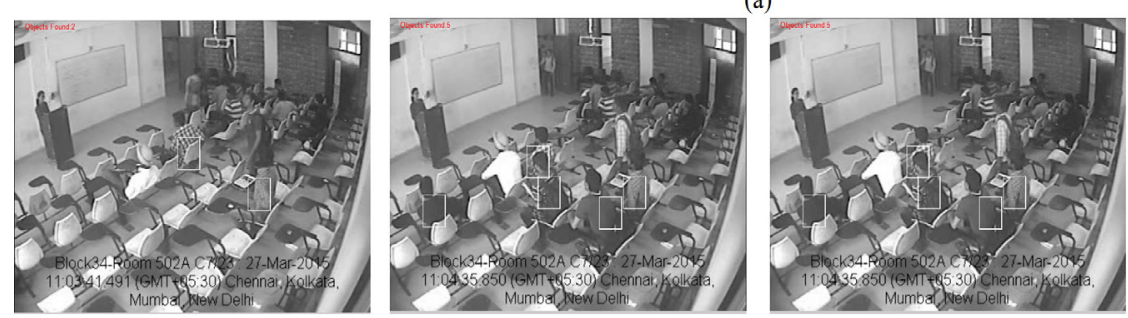

(b)
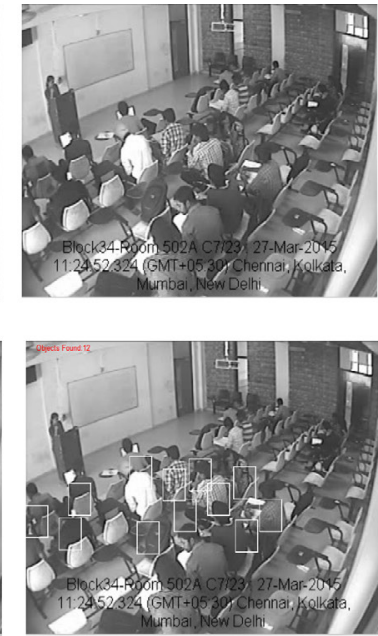

Figure 6. Foreground frames (1-4) of room number 34-502A before detection and after detection. (a) Before detection. (b) After detection.
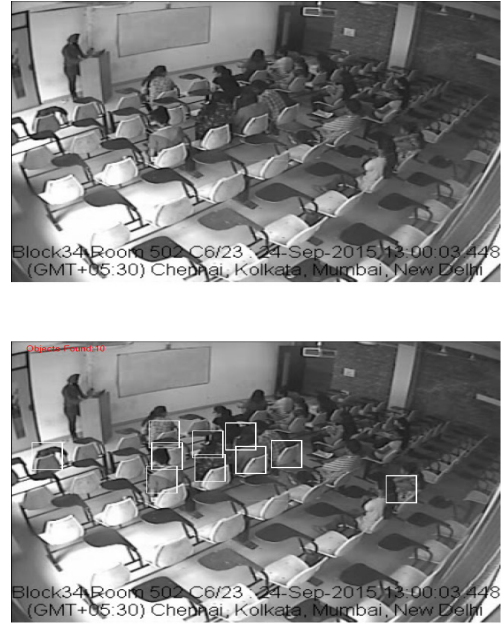

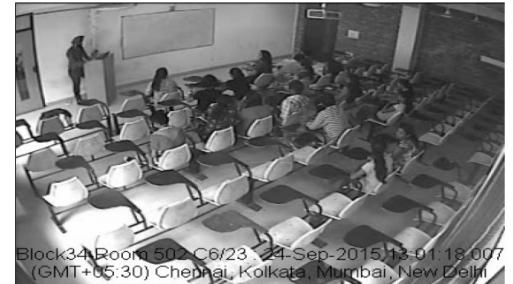

(a)

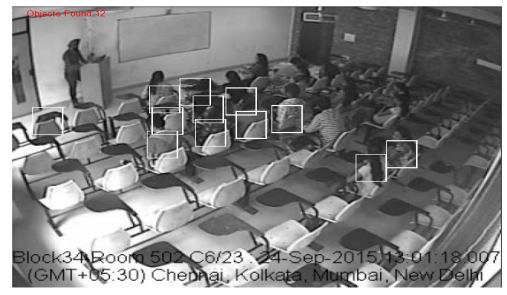

(b)
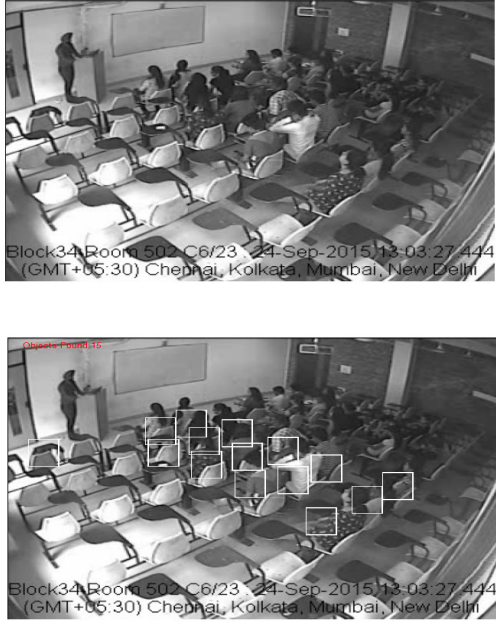

Figure 7. Foreground frames (1-3) of room number 34-502 before detection and after detection. (a) Before detection. (b) After detection.

Table 1. Accuracy, false negative, false positive and detection results by Our Proposed Approach over different frames of class rooms

\begin{tabular}{lcccccc}
\hline Frame No. & TP & APS & DS & FN & FP & Accuracy \\
\hline 34-502A_1 & 20 & 3 & 2 & 1 & 0 & $95 \%$ \\
34-502A_2 & 20 & 6 & 5 & 1 & 0 & $95 \%$ \\
34-502A_3 & 20 & 6 & 5 & 1 & 0 & $95 \%$ \\
34-502A_4 & 20 & 11 & 12 & 2 & 3 & $75 \%$ \\
34-502_1 & 30 & 9 & 10 & 2 & 3 & $86.6 \%$ \\
34-502_2 & 30 & 10 & 12 & 1 & 3 & $86.6 \%$ \\
34-502_3 & 30 & 13 & 15 & 0 & 2 & $93.3 \%$ \\
\hline
\end{tabular}

Table 2. Accuracy, false negative, false positive and detection results by Color processing of RGB planes over different frames of class rooms

\begin{tabular}{lcccccc}
\hline Frame No. & TP & APS & DS & FN & FP & Accuracy \\
\hline 34-502A_1 & 20 & 3 & 3 & 1 & 1 & $90 \%$ \\
34-502A_2 & 20 & 6 & 9 & 1 & 4 & $75 \%$ \\
34-502A_3 & 20 & 6 & 9 & 1 & 4 & $75 \%$ \\
34-502A_4 & 20 & 11 & 14 & 2 & 5 & $65 \%$ \\
34-502_1 & 30 & 9 & 10 & 2 & 3 & $86.6 \%$ \\
34-502_2 & 30 & 10 & 13 & 1 & 4 & $83.3 \%$ \\
34-502_3 & 30 & 13 & 16 & 0 & 3 & $90 \%$ \\
\hline
\end{tabular}


Table 3. Accuracy, false negative, false positive and detection results by Traditional Background Subtraction over different frames of class rooms

\begin{tabular}{lcccccc}
\hline Frame No. & TP & APS & DS & FN & FP & Accuracy \\
\hline 34-502A_1 & 20 & 3 & 4 & 1 & 2 & $85 \%$ \\
34-502A_2 & 20 & 6 & 11 & 1 & 6 & $65 \%$ \\
34-502A_3 & 20 & 6 & 11 & 1 & 6 & $65 \%$ \\
34-502A_4 & 20 & 11 & 12 & 4 & 5 & $55 \%$ \\
34-502_1 & 30 & 9 & 19 & 3 & 13 & $46.6 \%$ \\
34-502_2 & 30 & 10 & 19 & 1 & 10 & $63.3 \%$ \\
34-502_3 & 30 & 13 & 19 & 0 & 6 & $80 \%$ \\
\hline
\end{tabular}

Comparison of false positive, false negative and accuracy of our proposed approach with others techniques are shown in Figures 8, 9 and 10 respectively.

The attendance matrix for class rooms $34-502 \mathrm{~A}$ is shown in Figure 11 and for class room 34-502 is shown in Figure 12. The light green color shows the present students in the class room. First row shows the roll numbers of each student. The dark green color shows detection results of different approaches after execution. We are taking 20 places for class room 34-502A and 30 for 34-502. Attendance matrix also shows comparison of our

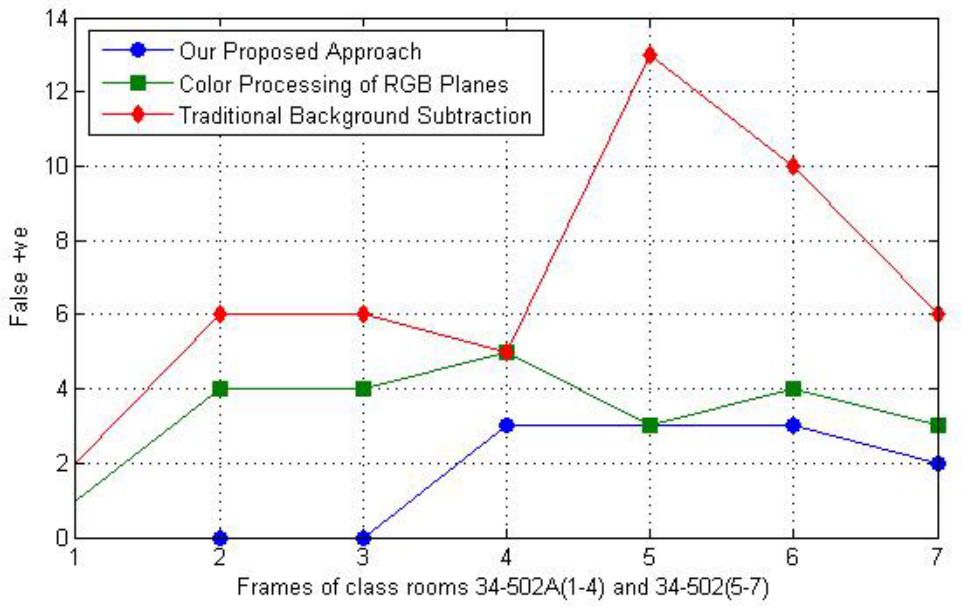

Figure 8. False positive comparison of our proposed approach with color processing or RGB planes and background subtraction.

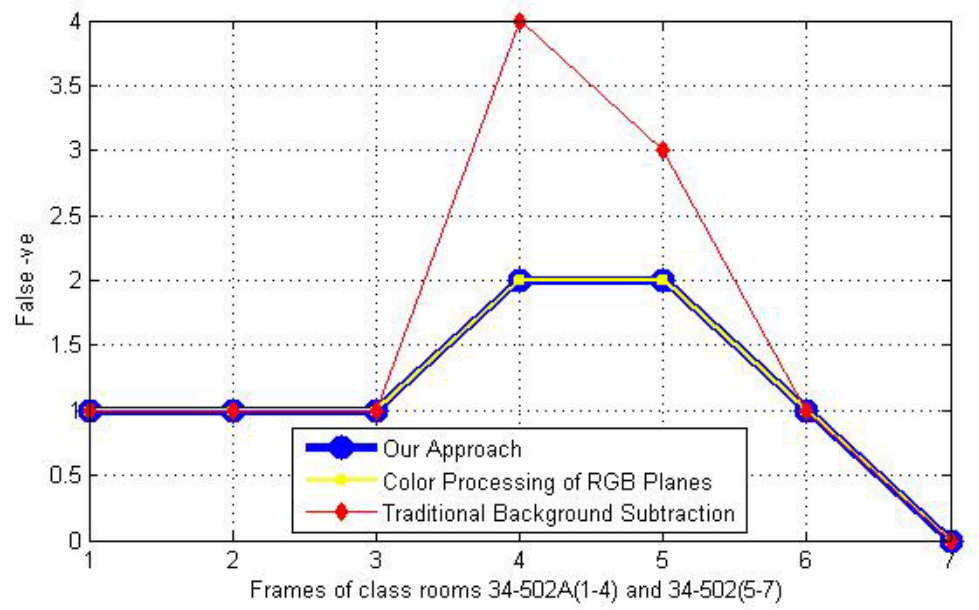

Figure 9. False negative comparison of our proposed approach with color processing or RGB planes and background subtraction. 


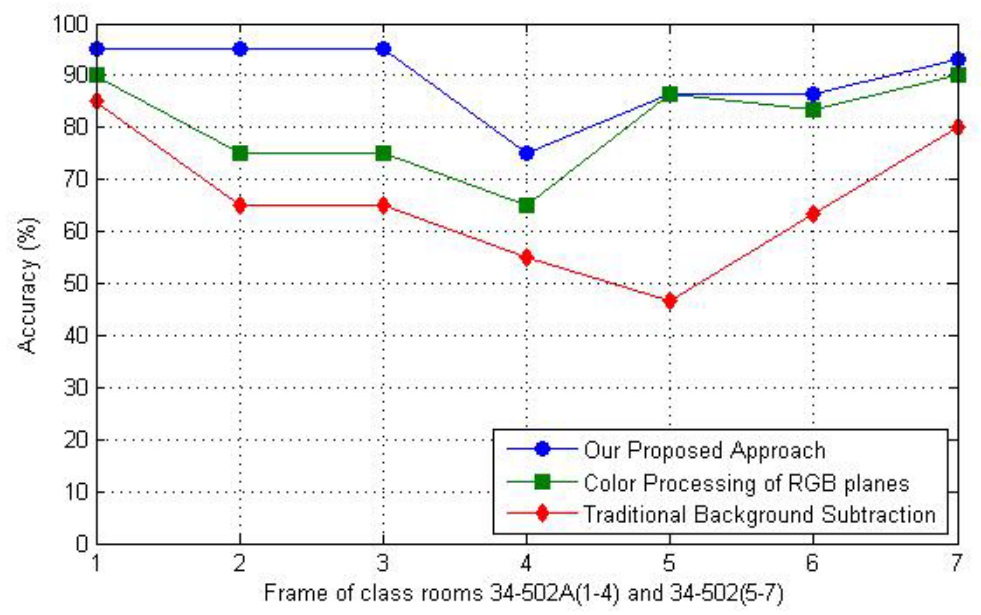

Figure 10. Comparison accuracy of our proposed approach with color processing or RGB planes and background subtraction.

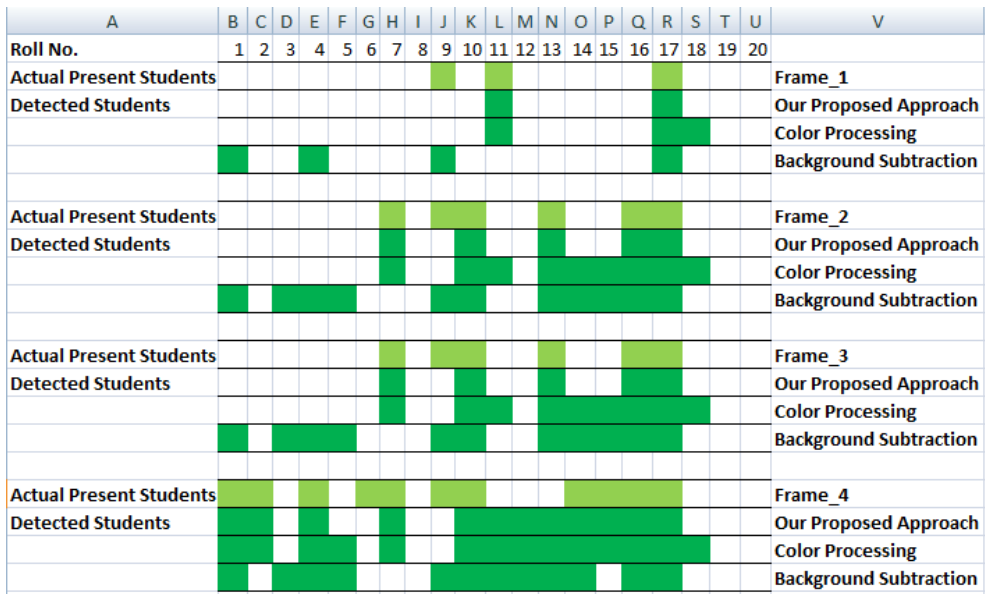

Figure 11. Attendance matrix of class room 34-502A.

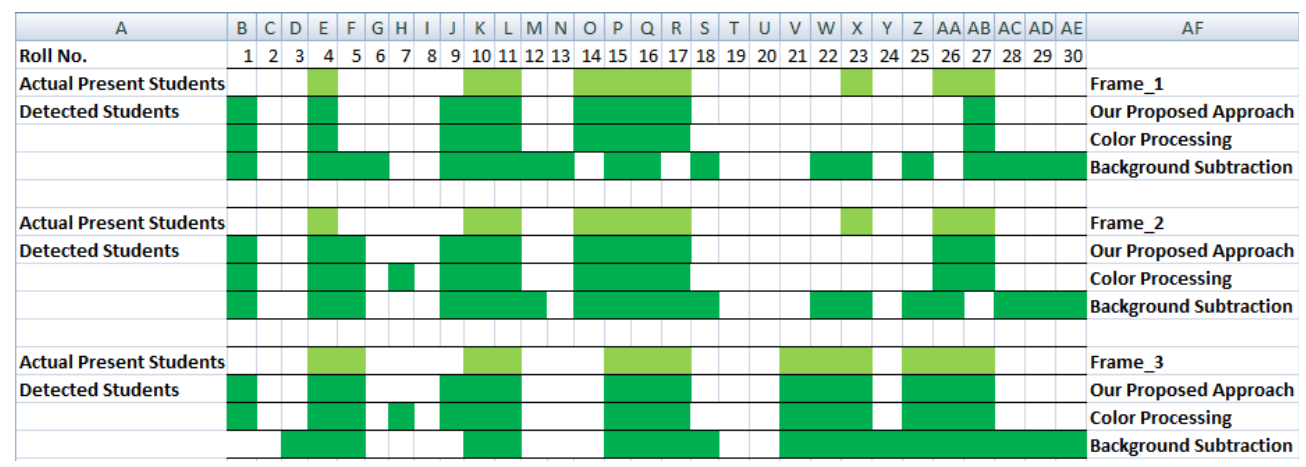

Figure 12. Attendance matrix of class room 34-502. 
proposed approach with color processing of RGB planes and background subtraction over different frames.

\section{Conclusion}

This report proposed an automated attendance system having some kind of artificial intelligence which will automatically takes the attendance of the students sitting in the class-room. It will also increase the performance and decrease the time interval for taking the attendance even if the strength of the class lies between 40-50 or more than fifty. Performance will increase in the sense that there will be no chance of marking the wrong attendance and also, student will not be able to shout the proxy for their friends. The new attendance system will automatically recognize the students sitting on the bench and mark them presents and others will be marked absent.

The results show that our proposed approach has more accurate as compared to color processing of RGB planes and traditional background subtraction. False Negative rate of our approach and color processing has same value but in case of false positive rate our proposed approach shows better results. Moreover, our proposed approach has less false negative as well as less false positive rate as compared to background subtraction approach. The proposed approach is also able to count the total number of objects present in the room. We have also tried to automatically detect the chairs based upon regular repeated pattern by using Automatic ROI detection algorithm. But still, we have not got $100 \%$ results. So, we have used pre-defined ROIs for proposed detection algorithm.

In the future, we will modify our Automatic ROI detection algorithm for getting 100\% results and used these automatic detected ROIs for the proposed detection algorithm and will also try to further refine proposed detection algorithm for more accurate results. We will also try to classify the different objects like human or bag present in the class room using object classification techniques which will further increase the accuracy of our proposed approach.

\section{References}

1. Khurana K, Awasthi R. Techniques for object recognition in images and multi-object detection. International Journal of Advanced Research in Computer Engineering and Technology. 2013 Apr;2(4):1383-88.
2. Hussin R, Juhari MR, Kang NW, Ismail RC, Kamarudin A. Digital image processing techniques for object detection from complex background image. Procedia Engineering. 2012; 41:340-4.

3. Dharani S, Gowri S, Ramya S. Human segmentation using Haar-classifier. International Journal of Engineering Research and Applications. 2014 Jul; 4(7):89-93.

4. Tareque $\mathrm{MH}, \mathrm{Al}$ Hasan AS. Human lips-contour recognition and tracing. International Journal of Advanced Research in Artificial Intelligence. 2014; 3(1):47-51.

5. Sujatha B, Santhanam T. Classical flexible lip model based relative weight finder for better lip reading utilizing multi aspect lip geometry. Journal of Computer Science. 2010; 6(10): 1065-9.

6. Sreenivas DK, Reddy CS, Sreenivasulu G. Contour approximation of image recognition by using curvature scale space and invariant-moment based method. International Journal of Advances in Engineering and Technology. 2014 May; 7(2):359-71.

7. Anam S, Uchino E, Suetake N. Image boundary detection using the modified level set method and a diffusion filter. Procedia Computer Science. 17th International Conference in Knowledge Based and Intelligent Information and Engineering Systems. 2013; 22:192-200.

8. Leordeanu M, Sukthankar R, Sminchisescu C. Generalized boundaries from multiple image interpretations. IEEE Transactions on Pattern Analysis and Machine Intelligence. 2014 Jul; 36(7):1312-24.

9. Bagherpour P, Cheraghi SA, Mokji MBM. Upper body tracking using KLT and Kalman filter. Procedia Computer Science. Proceedings of the International Neural Network Society Winter Conference. 2012; 13:185-91.

10. Ikizler N, Cinbis RG, Duygulu P. Human action recognition with line and flow histograms. 19th International Conference on Pattern Recognition; 2008. p. 1-4.

11. Kermani E, Asemani D. A robust adaptive algorithm of moving object detection for video surveillance. EURASIP Journal on Image and Video Processing; 2014. p. 1-9.

12. Yang JB, Shi M, Yi QM. A new method for motion target detection by background subtraction and update. Physics Procedia. International Conference on Medical Physics and Biomedical Engineering. 2012; 33:1768-75.

13. Maini R, Aggarwal DH. Study and comparison of various image edge detection techniques. International Journal of Image Processing. 2013;1(3):1-12.

14. Guo L, Liao Y, Luo D. Generic object detection using improved gentleboost classifier. Physics Procedia. International Conference on Solid State Devices and Materials Science. 2012; 25:1528-35.

15. Ma Y, Wu W, He Q. Algorithm for object detection using multi-core parallel. Physics Procedia. International Conference on Medical Physics and Biomedical Engineering. 2012; 33:455-61.

16. Aydin D, Ugur A. Extraction of flower regions in color images using ant colony Optimization. Procedia Computer Science. 2011; 3:530-36. 
17. Yue Y, An Z, Wu H. Adaptive targets-detecting algorithm based on LBP and background modeling under complex scenes. Procedia Engineering. 2011; 15:2489-94.

18. Dash A, Kanungo P, Mohanty BP. A modified gray level co-occurrence matrix based thresholding for object background classification. Procedia Engineering. International Conference on Communication Technology and System Design. 2012; 30(2011):85-91.

19. Wang L, Yung NH. Crowd counting and segmentation in visual surveillance. 16th IEEE International Conference on Image Processing. 2009; 4:2573-76.

20. Zhao L, Davis LS. Closely coupled object detection and segmentation. Proceedings of the Tenth IEEE International Conference on Computer Vision. 2005; 1:454-61.
21. Wu B, Nevatia R. Tracking of multiple, partially occluded humans based on static body part detection. Proceedings of the IEEE Computer Society Conference on Computer Vision and Pattern Recognition. 2006; 1:951-8.

22. Reddy MR, Vaithiyanathan V, Karthikeyan B, Venkatam TG. Change detection in video using pixel based parametric analysis. Indian Journal of Science and Technology. 2015; 8(35):5-9. DOI: 10.17485/ijst/2015/v8i35/80061.

23. Mehdi N, Ghodrat S, Amin E, Amanollah A. A new skin color detection approach based on Fuzzy expert system. Indian Journal of Science and Technology. 2015; 8(21):1-11. DOI: 10.17485/ijst/2015/v8i21/50606. 